\title{
Canta Cardenio, canta. Un ovillejo de ida y vuelta
}

\author{
Sing, Cardenio, sing. A round trip ovillejo
}

\author{
María Ángeles Ferrer Forés \\ Premio Acción Magistral FAD-Unesco \\ info@maferrerfores.com
}

Juan Francisco de Dios Hernández

Universidad Autónoma de Madrid juan.dedios@uam.es

\begin{abstract}
RESUMEN
Cervantes - de la Vega - Esnaola - Barbieri. Cuatro pilares sobre los que desenredar la estructura musical del Ovillejo quijotesco, una forma literaria ya de por sí sonora que tuvo una singular recepción en el catálogo musical cervantino. Esta investigación quiere presentar un trabajo analítico comparado desde el cual poder valorar elementos estéticos significativos del arranque de la música nacionalista tanto argentina como española. Tanto Barbieri como Esnaola coincidirán en las posibilidades musicales del Ovillejo cervantino desde la adaptación realizada por el dramaturgo Ventura de Vega. Encontraremos una resolución técnica diferente que define en buena medida las claves estéticas de una incipiente identidad sonora nacionalista que toma como centro y pretexto de acción la novela cervantina
\end{abstract}

Palabras clave: Canción, Teatro, Nacionalismo, Literatura, Romanticismo, Ovillejo.

\begin{abstract}
Cervantes - de la Vega - Esnaola - Barbieri. Four pillars to solve the musical structure of Ovillejo in Don Quijote, a literary form musical by itself, which had a unique success in Cervantes musical catalogue. This research is focused on a comparative analytical process from which we could stand out a common aesthetical beginning for Spanish and Argentinian Nationalist Music. Both Barbieri and Esnaola agreed about the musical possibilities of the same Ovillejo version due to the writer Ventura de la Vega. We will find different technical resolutions that define that common aim. The same Ovillejo offers a great chance to collate how both of them, using the same adaptation from Cervantes novel, founded their own national music.
\end{abstract}

Key words: Song, Theatre, Nationalism, Literature, Romanticism, Ovillejo. 


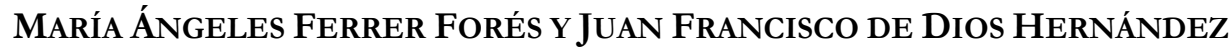

Ferrer Forés, M. Á.; De Dios Hernández, J. F. (2017). Canta Cardenio, canta. Un ovillejo de ida y vuelta. Cuadernos de Investigación Musical, 2, 59-73.

doi: http://dx.doi.org/10.18239/invesmusic_2017.02.1549

\section{El OVILLEJO COMO FUENTE MUSICAL}

El recorrido histórico del ovillejo o los ovillejos, tal como apunta Antonio Alatorre (Alatorre, 1990: 643-674) ${ }^{1}$, apenas se remonta a un tiempo inmediatamente anterior a Cervantes. Se trata de una forma o estructura poética que según la RAE presenta un esquema de versos octosílabos a modo de pregunta con un pie quebrado inmediato de respuesta que queda rematado con una redondilla final. Sin duda esta definición está planteada partiendo del Ovillejo que canta Cardenio en el capítulo 27 de la primera parte de El ingenioso bidalgo don Quijote de la Mancha. Y decimos canta, pues pese a que la RAE no presenta referencia alguna al respecto, Cardenio elabora una canción en tres coplas y tres redondillas. Alatorre analiza la intervención afirmando que la voz de Cardenio no es la voz de un pastor, sino una voz educada, "quien canta es un gran solista". A esto debemos añadir que Cervantes presenta a Cardenio cantando solo, sin acompañamiento alguno, un ejemplo prematuro de voz sola o a capela. Estamos, por tanto, ante un ejemplo poético eminentemente musical, que se desarrolla en virtud del ritmo natural que condiciona la propia forma junto a los aspectos de explosión de sentimientos que por aquel entonces triunfaban ya en Italia en forma operística. ¿Estamos ante un madrigal o una aria a voz sola?

Pese a que existen algunos ovillejos inmediatamente anteriores al cervantino (Alatorre, 1990: 643-674), es significativo que Cervantes opte por uno declaradamente cantado, algo que no se repetirá ni en el escritor alcalaíno ni en los posteriores ovillejos que nos conducen hasta el siglo XIX. Sin duda, el fundamento musical del ovillejo es claro de por sí. Observamos dos tempos bien definidos, lo que conduce de forma clara a una estructura bipartita. La clave musical es más bien rítmica, basada en la prosodia natural de las dos últimas sílabas de cada verso que se redondean con su repetición en el pie quebrado: “...mis bienes, desdenes.. / . ..mis duelos, los celos... / . . ausencia / paciencia...” No ya solo hablamos de un aspecto puramente musical sino que cabe añadir, por tanto, la importancia fundamental del texto, elemento que le aleja de unas canciones pastoriles que apenas suponían más que un pretexto para el uso de palabras musicales sin profundidad de mensaje.

Todos estos elementos nos deberían llevar a considerar este episodio del Quijote como uno de los más musicalizados desde su publicación. Pero curiosamente, quizá por la propia condición sonora del mismo, quizá porque condicionaba el texto hacia el género de

\footnotetext{
${ }^{1}$ Alatorre en su artículo presenta dos términos que utilizaremos en nuestra investigación. El primero es una conclusión sacada desde la voz de Ovillejo del DRAE, donde se define al ovillejo como "una secuencia poética de tres versos octosílabos, cada uno con respuesta en pie quebrado y una redondilla". Teniendo en cuenta esta definición la presencia de tres ovillejos permite el uso del plural. Por otro lado, Alatorre asume el uso del singular y define cada sección como copla.
} 


\section{CANTA CARDENio, CANTA. UN OVILLEJO DE IDA Y VUELTA}

la canción, no son muchos los ejemplos con identidad individual. Queremos decir con esto que existen ovillejos insertos en obras escénicas, sobretodo en óperas de los siglos XVII y XVIII, aunque hemos de esperar a la consolidación del género canción para encontrar más ejemplos.

Precisamente dentro de este género, tan marcado por el salón romántico, encontramos dos ejemplos de ovillejos que comparten elementos tanto externos como internos. Nos referimos a la canción "Quien mejora mi suerte", de Juan Pedro Esnaola escrita en $1839^{2}$ y el ovillejo de 1861 de Francisco Asenjo Barbieri ${ }^{3}$ inserto dentro de la obra teatral Don Quijote en Sierra Morena (escrita en 1831, estrenada en 1832), de Ventura de la Vega.

\section{Ventura de la Vega, TeXto PaRA UN QUiJOte MUSICAL}

Buenaventura de la Vega, conocido como Ventura de la Vega (Buenos Aires, 1807 Madrid, 1866), es sin duda alguna el personaje que amalgama esta investigación. Es el hilo conductor que reúne dos obras con un soporte textual semejante y con unas soluciones sonoras que, gracias al análisis comparativo, nos permiten alcanzar una serie de conclusiones sobre el proceso de identidad musical nacionalista en mitad del siglo XIX. Ventura de la Vega fue el pretexto para que dos compositores como Esnaola y Barbieri se embarcasen en un proyecto similar.

El argentino Buenaventura de la Vega había llegado a Madrid en plena adolescencia para recibir una educación española pese a que en aquel momento la identidad nacional argentina peleaba por definirse política y culturalmente. Las razones del viaje no difieren de las esgrimidas por el propio Juan Pedro Esnaola en el mismo año y con edades semejantes. La situación inestable de un país en pleno proceso de independencia instó a que ambas familias considerasen que España sería un lugar más fiable para educar a ambos adolescentes, habida cuenta de que igualmente provenía de ascendencia española. La presencia de Ventura de la Vega en el flamante Madrid de aquellos años le llevará a convertirse en un muy activo defensor de las vías modernas en el arte teatral del momento. Al contrario que su coetáneo Juan Pedro Esnaola, de la Vega opta por continuar en Madrid

\footnotetext{
${ }^{2}$ La canción, cuyo título original es literal, aparece enmarcada en una colección de canciones sin un concepto definido de ciclo bajo el título de Colección de Canciones con acompañamiento de Piano-Forte.

${ }^{3}$ Llama la atención y nos define ya la fascinación del propio Barbieri por el ovillejo, que en su juventud con apenas 21 años, escribiera unos ovillejos muy en consonancia con los cervantinos, aunque de ritmo considerablemente más torpe:

"Niña, es tu faz. cual la rosa

bermosa;

tienen tus ojos duliura

pura;

eres tierna tortolilla

sencilla;

Dime pues, flor de Castilla

cómo pretendes no muera

de amor, al verte hechicera

hermosa, pura y sencilla..."
} 


\section{MARÍA ÁNGELES FERRER ForÉS Y JUAN FranCisCo DE DiOS HERNÁNDEZ}

desarrollando su talento literario y desde entonces comenzará una carrera llena de reconocimiento y capacidad decisoria en la cultura de la corte.

Con apenas 25 años de edad, el joven de la Vega estrenó en el Teatro de la Cruz la obra Don Quijote en Sierra Morena, obra cuya proyección final parecía ser la de servir como texto para una ópera o zarzuela. La obra no tuvo una gran repercusión, si bien María José Rodríguez Sánchez de León afirma que la obra se ajustará al programa de definición del nuevo teatro español fernandino y que esta posición privilegiada le granjeará numerosos beneficios personales y profesionales (Rodríguez Sánchez de León, 2006: 209-220).

Es bien sabido que la obra no gozó de un gran éxito, si bien todas las críticas recibidas por de la Vega presentaban siempre la condescendencia del colega hacia un joven y prometedor dramaturgo. De cualquier modo, el texto de 1832, con sus posibles errores señalados por sus colegas, no se llegó a editar, de modo que un texto llamado a ser referente de una tendencia incipiente no pudo llegar a tanto. En el propio manuscrito original de 1832, Ventura de la Vega planteaba que la obra podía admitir una musicalización, seguramente en forma de zarzuela, que por entonces comenzaba a abrirse camino como vehículo para el nuevo género nacional. No hemos de perder de vista este hecho de cara a encontrar una justificación plausible por la que el Ovillejo escrito por Ventura de la Vega aparecerá en una canción de salón argentina casi siete años después del estreno de la obra teatral.

El devenir de Don Quijote en Sierra Morena conocerá una segunda oportunidad dentro de los festejos periódicos que desde 1861 la Real Academia de la Lengua Española decide poner en marcha para celebrar la figura y la obra de Miguel de Cervantes Saavedra. Ventura de la Vega era por entonces miembro de la Academia y su carrera había crecido de forma exponencial. Por su parte, Francisco Asenjo Barbieri, a la sazón único músico admitido como miembro de derecho en la Real Academia tres décadas después, será una figura de creciente importancia desde ese momento en la institución. La relación entre ambos intelectuales es bien conocida y en algunos momentos incluso estrecha, llegando a la amistad. Esta colaboración se había fraguado ya en la década anterior con la colaboración de literato y músico en varias zarzuelas. No es de extrañar por tanto que Ventura de la Vega, desde su posición en la Academia, propusiese al mismo Barbieri para asumir un papel importante en estos festejos cervantinos. Tras unos primeros intentos por definir el tipo de homenaje musical que se pretendía cumplir desde la Academia, fue en 1864 cuando la programación musical, con cargo de maestro de capilla, recayó en Barbieri (Lolo, 2008: 391-403). En aquel mismo año de 1861, Barbieri recibió el encargo de redactar un plan de reforma para las enseñanzas en el Real Conservatorio de Música y Declamación de manos de su director, que no era otro que el propio Ventura de la Vega.

Con motivo de los primeros fastos organizados en 1861 se reestrenó Don Quijote en Sierra Morena, en versión revisada y reducida, en el Teatro del Príncipe madrileño. Con motivo de esta representación y cumpliendo con uno de los objetivos marcados por el propio dramaturgo, en aquella ocasión sí que contó con música, tal como aparece en la dedicatoria del texto revisado para la ocasión. Escribió Barbieri música en tres momentos 


\section{CANTA CARDENio, CANTA. UN OVILLEJO DE IDA Y VUELTA}

puntuales, el Ovillejo, para cuerda y tenor en el primer acto; el Bailete para orquesta en el segundo; y un Tempo de Marcha para tenor, coro masculino y orquesta en el tercero y último de los actos. Es interesante señalar que los medios con los que contó la obra fueron extraordinarios habida cuenta de que se pudiese contar con una orquesta, un coro y un solista para tres intervenciones puntuales. Además, cabe añadir que no son tres piezas fáciles, sino que requieren de un trabajo y unos ensayos importantes para tan poca presencia dentro de la obra general. Igualmente debemos considerar un hecho sobre el que plantearemos en su momento una tesis concreta. De las tres intervenciones realizadas por Barbieri, el Ovillejo no trabaja desde el texto de Ventura de la Vega, sino del propio Cervantes; respecto al Bailete es evidente su concepto instrumental y, finalmente, el Tempo de Marcha presenta un texto no relacionado con la obra ni con los personajes, sino a modo de Loa a Cervantes. ¿Qué pudo ocurrir para que el compositor no tuviera en cuenta los textos del dramaturgo en las aportaciones musicales a la obra teatral? Como ya hemos indicado, no se trataba de la primera colaboración entre ambos. No hemos de olvidar que en 1851, Barbieri y Ventura de la Vega firmaron una de las grandes obras maestras de la zarzuela romántica, Jugar con Fuego; en 1853, volvieron a colaborar en El Marqués de Caravaca y, finalmente, en 1867 el libro póstumo, El tesoro escondido ${ }^{4}$. A juzgar por la intricada génesis de una obra conjunta sobre Don Quijote que partiría, según las noticias que tenemos, de 1857, Barbieri ya tenía el Ovillejo compuesto anteriormente a los festejos cervantinos de 1861. El trato personal fue siempre muy bueno incluso en vida de ambos y después de la muerte del literato, Barbieri se enzarzó en polémicas con críticos y hombres de letras en defensa de Ventura de la Vega, a quien atribuía la autoría completa de la definición del género zarzuelístico sin contar con conocimientos musicales pero con una verdadera intuición lírica. La presencia testimonial de Barbieri en el Quijote de 1861 no es óbice para que de la Vega se mostrase muy contento en la dedicatoria refiriéndose a la música compuesta por Barbieri como un "adorno".

\section{Juan Pedro Esnaola, ovillejo de ida}

Pese a lo que pudiera parecer, la historia de Juan Pedro Esnaola y el Quijote enlaza a la perfección con los datos que hemos venido desgranando. Juan Pedro Esnaola (Buenos Aires, 1808-1878), pese a ser uno de los pilares de la primera música argentina, bien por su recorrido personal, bien por la elección de un tipo estético europeizante, no es un compositor que posea una consideración importante ni respaldo nacional o internacional. Valgan las palabras del musicólogo Bernando Illari, especialista en la vida y obra de Esnaola: "La imagen que las historias de la música proyectan de Esnaola tiene poco que ver con su antigua fama. No se lo cree compositor hecho y derecho, sino meramente precursor de otros que sí serían compositores completos y argentinos genuinos. En tanto compositor solo a medias, carece de una línea propia" (Esnaola, 2009). Illari defiende la importancia de Esnaola como compositor pues le atribuye las bases para una posterior consolidación de las identidades musical argentinas. Hemos de tener en cuenta que el amanecer del nacionalismo hispanoamericano, que se produce políticamente con las sucesivas

4 También es reseñable que el propio Barbieri colaboró con el hijo de Ventura de la Vega, Ricardo de la Vega (1839-1910) en sus últimas obras. 


\section{MARÍA ÁNGELES FERRER ForÉS Y JUAN FranCisCo DE DiOS HERNÁNDEZ}

independencias de la metrópoli española, posee unas características no tan diferentes al resto del continente desde el punto de vista cultural. El desarrollo de los autogobiernos consolida a una pequeña oligarquía naturalmente cultivada y no autóctona que continua en cierto modo, o imita en la mayoría de las ocasiones, los usos y costumbres de sus émulos europeos. Es por ello que buena parte de los compositores, al igual que en el gremio político, están formados si no en Europa, sí de acuerdo con los gustos europeos. Esta mímesis suele llamar a error respecto del nacionalismo americano (proceso idéntico al hispano se produce en Norteamérica), que se considera tardío pues tan solo tiene en cuenta la presencia de elementos autóctonos o indígenas en forma de ritmos, melodías o temáticas que irrumpen a fines del XIX y, sobre todo, en la primera mitad del siglo XX. Al igual que los nacionalismos europeos, sus definiciones estéticas se abren paso con lentitud desde los marcos socioculturales centroeuropeos románticos y este extremo se repite de forma clara en la música hispanoamericana del XIX.

Esnaola no es una excepción en este proceso de mímesis del salón y sus inercias. Pese a que es un niño durante el proceso de independencia, su viaje iniciático se produce con tan solo 10 años, en 1818, junto a su tío José Antonio Picasarri, guipuzcoano y maestro de capilla en la Catedral de Buenos Aires. Es más que probable que como otros muchos, Picasarri saliese del país al no estar dispuesto a renunciar a su nacionalidad española. Siendo el padre de Esnaola español y su madre criolla, su presencia en España durante aquellos años volátiles bonaerenses tiene también algo de protección frente a los tiempos inciertos. La formación musical de Esnaola no parece mostrar ningún talento especial, pues sus estudios pianísticos y de composición los hace de forma privada entre Madrid y París. No existen demasiados datos sobre sus movimientos en Europa, pero sí es cierto que aprenden a la perfección los gustos del salón europeo que serán su seña de identidad posterior. Madrid y París serán sus lugares de formación, frecuentando los salones del momento y aprendiendo de un concepto por entonces pujante que definía de forma clara las nuevas tendencias estéticas del romanticismo.

Esnaola regresa a Buenos Aires con 14 años, en 1822, y ya en la década de 1830 forma parte activa de la vida musical bonaerense. Su vertiente musical es secundaria durante este período, habida cuenta que Esnaola pasó por ser un "comerciante exitoso y amasó una fortuna” (Esnaola, 2009). Cuenta Illari, citando numerosas fuentes, que su fortuna se fraguó en la especulación y el alquiler, asignándole incluso la posesión de varios conventos. De modo que Esnaola a fines de la década de 1830 se presenta como músico de salón como algo sobrevenido, si bien ya en la siguiente década su papel como compositor le sitúa en un espacio diferente. Es en esta década de 1830 cuando comienza una labor compositiva no sistemática en la que se hace eco de las variables propias del salón europeo. Abundan en sus creaciones las canciones junto con los valses y demás pequeñas formas y estructuras. El carácter aficionado de Esnaola se muestra claro ante la ausencia de obras de gran calado formal o mayor ambición estética.

Las relaciones de Juan Pedro Esnaola con el régimen de Juan Manuel de Rosas, que tuvo lugar entre 1835 y 1852, fueron complejas de acuerdo con la historiografía al respecto. Aunque el Himno Nacional Argentino fue obra del murciano Blas Parera (Murcia, 1776 - 


\section{CANTA CARDENIO, CANTA. UN OVILLEJO DE IDA Y VUELTA}

Mataró, 1840), una de las adaptaciones musicales -las textuales fueron más habituales y traumáticas- se debió a Esnaola, que completó dicha propuesta armonizada entre 1847 y 1849 y su versión orquestal en 1860. Este hecho, junto al magisterio ejercido por el compositor con la hija del dictador Rosas, tildaron directamente a Esnaola como músico del régimen. Illari defiende no ya solo la independencia de Esnaola respecto de Rosas, sino incluso su condición de víctima en ciertos aspectos (Illari, 2010: 33-73). De cualquier modo, ya en la década de 1840 nos encontramos a un Esnaola compositor y maestro de muchos en la Escuela de Música y Canto de Buenos Aires, fundada en 1822 por su tío José Antonio Picasarri donde Esnaola ejerció la docencia desde muy joven.

Todos estos elementos biográficos nos indican que, pese a lo que la historiografía ha venido indicando, la formación y la creación musical de Esnaola poseen la solidez y proyección suficiente para hablar de un papel preeminente en el primer nacionalismo musical argentino. Las características de éste no difieren de las desarrolladas en otros países del entorno e igualmente en Europa. La falta de un tejido social e inmobiliario adecuado, impidió en la mayoría de los países americanos que la ópera fuese un motor estético. Quizá el caso español arroja más semejanzas a América que al resto de Europa, con la salvedad de las grandes ciudades del país. No hemos de olvidar que el tejido de teatros en España arranca tímidamente en la década de 1820 y que se consolida durante el reinado de Isabel II, extremo que se tiende a repetir en los países americanos cuya independencia se gestó en ese inicio del siglo XIX. De este modo, ante la ausencia obvia de espacios adecuados, el efecto transmisor del teatro musical como fuente de actividad estética y sociológica no se lleva a activar y con ello la ausencia de formaciones adecuadas para las funciones teatrales y por consiguiente de las primeras orquestas estables ${ }^{5}$. Las pequeñas formas instrumentales y sobretodo el lied o canción se convierten en estandartes del arranque del Romanticismo afín a Europa y, por imitación de los usos sociales, a la flamante América independiente.

En este entorno nos encontramos con un joven Juan Pedro Esnaola que se convierte en el compositor de cabecera de la sociedad bonaerense de mediados de la década de los 30. Su creación se centrará por tanto en la música de salón, componiendo un gran número de pequeñas danzas de moda junto con canciones. Un elemento significativo será el uso en su mayor parte de textos de poeta argentinos. Uno de los primeros problemas que hemos de resolver llegados a este punto es por qué y de qué manera Esnaola se hace con el texto de Ventura de la Vega y lo incluye tiempo después dentro de su Colección de Canciones acompañadas con Piano-Forte ${ }^{6}$.

\footnotetext{
5 Tal como afirma Di Benedetto, 1987: 39-47, no hemos de olvidar que en la formación de las primeras orquestas estables, la mayoría de ellas aún en funcionamiento, se conforman primero dentro de los entornos de las Sociedades de Conciertos alemanas y sobreviven como orquestas residentes de los primeros teatros musicales en Centroeuropa, desarrollándose ya en la segunda mitad del XIX como formaciones para el repertorio sinfónico.

${ }^{6}$ La colección reúne canciones de poetas en su mayoría argentinos, con la inclusión del riojano Bretón de los Herreros y dos canciones en italiano junto a otras sin atribución textual, compuestas entre 1833 y 1841 . El propio compositor rubricó al final de cada una de las canciones con la fecha de composición. No podemos hablar de un ciclo teniendo en cuenta la ausencia de un hilo conductor argumental, pero sobretodo por lo dilatado de su gestación. Se trata por tanto de una colección realizada sin duda alguna a posteriori y que no ha sido editada. Pese a lo que reza el título, no todas las canciones presentan un acompañamiento para piano-
} 


\section{MARÍA ÁNGELES FERRER ForÉS Y JUAN FranCisCo DE DiOS HERNÁNDEZ}

Podemos especular con la posibilidad de que viajaran juntos hacia Europa en 1818, pues el año es coincidente, si bien carecemos de datos más exactos para afirmar que lo hicieron en las mismas fechas. Pero no es menos cierto que la lógica nos permite especular con la más que segura coincidencia de dos argentinos adolescentes en Madrid. Pese a que, como ya indicamos, Ventura de la Vega opta por no regresar, este hecho no es un impedimento para que años después, Juan Pedro Esnaola recupere un texto de Ventura de la Vega para completar una canción.

Tal como aparece en el manuscrito original, la canción fue escrita el 10 de mayo de 1839, siendo una de las últimas de la colección. Posteriormente, Esnaola reunió las composiciones vocales de esta época en un volumen con 48 canciones que se desarrollan dentro de una estética claramente romántica aplicando los parámetros armónicos y temáticos del primer romanticismo europeo. Teniendo en cuenta que el Ovillejo o Canción de Esnaola está enmarcada en una hipotética colección en la que prevalece el uso de textos de escritores argentinos, la presencia de Ventura de la Vega no desentona, sino que incluso se presenta como un elemento novedoso pues utilizaba un texto de una obra no circunscrita al género del poema, sino al teatro o a la novela.

Esnaola podría haber utilizado el texto original de Cervantes, pero opta por usar el texto de Ventura de la Vega citado perfectamente en el encabezamiento de la composición. Respecto del texto cervantino, Ventura de la Vega afrontó el ovillejo con una clara aspiración de actualizar dicho texto, procurando con ello eliminar elementos que podrían caer en el anacronismo. De la Vega mantiene evidentemente la estructura de tres versos octosílabos, cada cual con su pie quebrado, y la redondilla de remate, pero reduce las coplas de tres (la cervantinas originales) a dos. En este caso asiste una razón de tiempo teatral que, obviamente, no tenía Cervantes en una novela, aunque su natural indica que fuese escrita para leer a viva voz ${ }^{7}$. Pese a todo, llama la atención que Ventura de la Vega optase por prescindir de la primera de las coplas, que por motivos obvios resulta la más conocida y famosa, centrándose en las dos coplas siguientes. Esto nos introduce en una de las grandes divergencias textuales, pues Esnaola, pese a citar expresamente que el texto de su compatriota, cambia el orden en las coplas e incluso altera el tiempo verbal del primero de los versos.

Llama la atención desde el principio el cambio de orden en las dos coplas. Existe en Esnaola el interés por comenzar de manera más brillante y sobretodo cercana al original, es por ello que respetando que Ventura de la Vega prescinde de la primera copla, Esnaola altera la copla de inicio (tercera desde el original) en incluso pasa del mejorará inicial al mejora que elige como inicio. Teniendo en cuenta que Cervantes usa el futuro simple, se nos abren dos posibilidades para aclarar esta divergencia. Por un lado, que Esnaola considera que para

forte (denominación que en las últimas obras se reduce ya a piano) sino que hay una para arpa y la que nos ocupa para guitarra. Todo eso nos devuelve al razonamiento inicial de que no se trata de un como tal.

${ }^{7}$ La transmisión de los textos de novelas y teatro a viva voz ha sido ampliamente estudiado por Chevalier o Bennassar dentro de los usos culturales de la Edad Moderna. Nosotros nos hemos basado en el artículo de Paz Gago, José María, Oralidad, escritura y visualidad en el Quijote, en Asociación Internacional del Siglo de Oro, Actas, 1996, obtenido en los fondos informatizados del Centro Virtual Cervantes, publicadas por María Cruz García de Enterría y Alicia Cordón Mesa, Alcalá de Henares 1998.

Cuadernos de Investigación Musical, 2017, junio, n² 2, págs. 59-73.

ISSN: 2530-6847

DOI: http://dx.doi.org/10.18239/invesmusic_2017.02.1549 


\section{CaNta Cardenio, CANTA. Un OVILlejo DE IDA Y VUELTA}

su melodía una sílaba más altera el tempo; por otro, cabría la remota posibilidad de que existiese una errata en el texto utilizado por Esnaola.

Poder avanzar la teoría de que Esnaola, compositor casi aficionado, utiliza los textos la libertad de alguien que no intuye proyección cualitativa alguna más allá de una pequeña canción de salón. Esta propuesta se satifica si observamos que en la redondilla que cierra la copla inicial en Esnaola, no se respeta el primer verso del escritor: "... De este sueño no es cordura..." optando por "... De este modo no es cordura..." que en realidad es el verso original del Cervantes con la única anomalía de "este" por "ese". Esnaola trabaja por lo tanto con ambos textos durante el proceso de creación, optando por la versión que mejor conviene a su línea de canto.

El resto de alteraciones son puntuales y responden a razones de actualización o hipotética corrección. En la primera copla de Ventura de la Vega (segunda en Esnaola), el dramaturgo comete un error ortográfico en el facsímil original y en el segundo verso aparece "repuna", algo que tanto en Cervantes como en Esnaola (que nuevamente corrige) se convierte en "repugna". El caso de la palabra "estraño" (segundo verso de la primera redondilla), de la Vega mantuvo el arcaísmo cervantino, mientras que Esnaola vuelve a corregir, en esta ocasión a ambos, y escribe "extraño".

Podemos concluir que en el capítulo textual, Juan Pedro Esnaola trabaja con ambos ovillejos (Cervantes y de la Vega) optando de forma indistinta por uno u otro en virtud de sus propios intereses. Además cabe añadir el hecho evidente de que Esnaola, aun manteniendo como autor a Ventura de la Vega, adapta el orden en un claro posicionamiento estético, manteniendo los fines musicales sobre los textuales.

Para adentrarnos en el terreno puramente musical debemos subrayar que la partitura de Juan Pedro Esnaola no ha sido editada como tal y que se ha trabajado desde una copia del facsímil original disponible en la red por parte de la Biblioteca Digital Trapalanda de la Biblioteca Nacional de Buenos Aires. ${ }^{8}$ Se ha realizado una edición ad hoc para solventar cuestiones de escritura y cotejar determinados elementos dentro del análisis.

Estamos ante una canción compuesta para vOz y guitarra ${ }^{9}$ en la que no se especifica el tipo de $\mathrm{voz}^{10}$. Por su ámbito Mi 3 (puntualmente desciende a un Re\# 3 tan solo con repercusión de apoyo) - Sol 4, presenta varias posibilidades, pese a que en su línea de canto y el tipo de ataques, parecería pensada para voces agudas antes que intermedias. La parte de guitarra presenta una textura en acordes arpegiados con sostén del bajo armónico funcionando como Bajo d'Alberti, muy en la línea de los acompañamientos guitarrísticos habituales en la primera mitad del $\mathrm{XIX}^{11}$. Su función es puramente gregaria sin

\footnotetext{
8 Recurso en línea: http://trapalanda.bn.gov.ar/jspui/handle/123456789/6046 [última consulta: 23 de agosto de 2016].

9 Junto con la canción El pensamiento inoportuno (1837) son las dos únicas de la colección no escritas para acompañamiento de pianoforte, en su caso particular el instrumento es el arpa.

${ }^{10}$ En toda la colección tan solo en la canzonetta italiana Addio se especifica la voz de tenor (tenore), no así en la otra canción en italiano, La partenza, donde mantiene la falta de definición vocal.

${ }^{11}$ Existen relaciones evidentes con las canciones españolas de moda en la época, como los casos de Manuel

García o Federico Moretti.
} 


\section{MARÍA ÁNGELES FERRER ForÉS Y JUAN FranCisCo DE DiOS HERNÁNDEZ}

protagonismo alguno pese a su presencia solista en el inicio, en los períodos entre estrofas y en la coda final.

La obra fue compuesta en un día, 10 de mayo de 1839, tal como aparece consignado al final de la partitura manuscrita, si bien esta cronología podía indicar el día de finalización. No existe un discurso unitario entre los musicólogos especialistas en la obra de Esnaola respecto a esta u otras obras. Sin título, sólo aparece Canción, la filiación del texto aparece en la esquina superior izquierda donde leemos: "De drama "D Quijote" de D. V. de la Vega.

La canción aparece en el folio $\mathrm{n}^{\circ} 53$ y vuelta de la serie de composiciones que Esnaola realiza entre 1838 y 1841, momento especialmente significativo en la vida del compositor. Consta de 41 compases con una estructura bipartita A-B con repetición de B. El tempo de la sección A es Larghetto, que se corresponde con las tres coplas y pie quebrado del texto, mientras que la Redondilla presenta la asignación de molto legato e dolce, sección B. Existe igualmente una introducción y una coda puramente armónicas que cubren los compases del 1-6 y del 38-41. Al final de la partitura aparece escrita la segunda de las coplas del ovillejo.

La obra presenta una armadura de un sostenido que responde a la tonalidad de mi menor. El comportamiento tonal es clásico, sin buscar efectos más allá de los recursos de la segunda napolitana. El uso de los acordes arpegiados le permite a Esnaola retrasar algunas notas lo que provoca cierto movimiento. La estructura formal también se ve influida por el planteamiento tonal, pues Esnaola juega con la tonalidad menor al inicio, coincidiendo con las coplas (pregunta-respuesta) y con la mayor, en este caso Sol Mayor para subrayar la redondilla de respuesta. El recurso es efectivo y adecuado para el fin y la función para la que está escrita la partitura, que no es otro la música de salón para consumo directo de la burguesía y aristocracia.

Desde un punto de vista motívico encontramos elementos propios de la prosodia musical operística belcantista. Esnaola opta por un motivo descendente para plantear la pregunta con una respuesta a modo de eco progresivamente más grave. El perfil es directo, efectivo y dramático. Por su parte, la redondilla, que funciona como respuesta global, presenta un trabajo más ondulante y preciosista apoyándose en el paso a la tonalidad mayor. Es interesante que Esnaola opte por repetir la redondilla de forma literal a nivel textual, pues denota unos usos compositivos muy europeizantes y muy influidos por el teatro musical italiano. También es digno de reseñar que el proceso de coda cadencial final no presenta indicaciones que se refieran a la repetición de la segunda copla excepto por la consignación escrita del texto. Se trata de un uso habitual que probablemente hubiera recibido el tratamiento lógico en caso de edición de la partitura.

Es por tanto una obra que cumple de forma clara con las variables de la música del primer romanticismo europeo al que le añade elementos deudores de la música española del momento. Como música de salón, presenta perfiles que definen a la perfección los recursos y tendencias habituales en el incipiente nacionalismo americano independiente que estéticamente procedía como émulo de las tendencias europeas. 


\section{CANTA CARDENIO, CANTA. UN OVILLEJO DE IDA Y VUELTA}

\section{BARBIERI, OVILLEJO DE VUELTA}

Bajo el título de Ovillejo, la génesis y el objetivo de la obra de Barbieri son bien diferentes a la canción de Juan Pedro Esnaola. Ya de entrada nos encontramos con un uso del término literario, algo que Esnaola no necesita. Encontramos por tanto un horizonte marcado por la excelencia en el proceso que sitúa a Barbieri en otro espacio creativo muy diferente. El proceso de justificación, proceso y creación ya fue analizado por Begoña Lolo, señalando que fue dentro de los actos de homenaje a Cervantes realizados por la Real Academia de la Lengua cuando se repuso la revisión de la obra Don Quijote en Sierra Morena, de Ventura de la Vega (Lolo, 2008: 391-404). Como ya indicamos la obra de 1832 no gozó de éxito de modo que para el escritor, la posibilidad de revisar y reestrenar su obra fue muy importante. Su relación con la Real Academia de la Lengua siguió los pasos habituales, siendo académico honorario en 1842, supernumerario dos años después y finalmente académico de número, ocupando la silla $\mathrm{F}$, en 1845. Su conocimiento de la Academia era, por lo tanto, importante y su capacidad de decisión ya debía ser grande en aquellos años.

Por su parte, Francisco Asenjo Barbieri (1823-1894) pasaba por ser un músico reputado y de una vasta cultura, algo extraño dentro del mundillo intelectual decimonónico. Su defensa de la lengua castellana como vehículo natural para el teatro musical nacional provocó no pocas controversias en la década de los 40 aún siendo muy joven. Este hecho sin duda le puso en primera línea. Su presencia en el entorno de la Academia era significativo ya en la década de los años 60 . Si bien es cierto que su entrada como miembro de la institución no se produjo hasta 1892, ocupando la silla $\mathrm{H}$, es evidente que la presencia de Barbieri era importante en las instituciones ${ }^{12}$ desde hacía algunas décadas.

La relación que debió de existir entre Ventura de la Vega y Francisco Asenjo Barbieri fue larga y afectuosa. La temprana muerte del escritor no fue impedimento para que Barbieri le recordase casi treinta años después de su muerte en su discurso de entrada en la Academia de la Lengua como “... mi inolvidable amigo y compañero Ventura de la Vega..." (Barbieri, 1892: 25). En dicho discurso, Barbieri elaboraba un brillante razonamiento sobre la musicalidad de la lengua castellana en la que era obligatoria la referencia cervantina y al Quijote. No cabe duda por tanto que Barbieri colaboraba de forma habitual con la Academia y que por tanto su presencia en los fastos cervantinos de 1861 no era algo noticiable pese a la temprana edad del compositor.

La génesis de una zarzuela quijotesca se remonta a la década anterior. Las colaboraciones entre ambos habían producido grandes títulos y beneficios para ambos. En una carta escrita en verso por Barbieri a Ventura de la Vega en 1857 podemos leer ya una clara referencia a un proyecto inminente: “...iEs posible que arrincones/ al de la triste figural dejándome al par más tristel que noche de espesa bruma?..." (Casares, 1994: 167). El proyecto comenzó a tomar forma en 1859, cuando Ventura de la Vega inició por fin la redacción de un libreto sobre El Quijote, a buen seguro recuperando y adaptando su obra de 1832. Barbieri afirma que ya había comenzado la composición de la obra: "... Ventura de la Vega

\footnotetext{
${ }^{12}$ Es interesante señalar que Barbieri será en encargado de leer el discurso de introducción de la sección de Música dentro de la Real Academia de Bellas Artes de San Fernando en 1874, abriendo camino con ello un año antes a una avalancha de compositores en la institución.
} 


\section{MARÍA ÁNGELES FERRER ForÉS Y JUAN FranCisCo DE DiOS HERNÁNDEZ}

empezó a escribirme una zarzuela sobre El Quijote, de la cual ya tenía puesta en música dos piezas cuando parece que fue a verle Adolfo García y le rogó que no continuase porque él tenía ya concluida esta obra sobre el mismo asunto ${ }^{13} . . " 14$ (Casares, 1994: 210). Este intento fallido de zarzuela había permitido a Ventura de la Vega revisar su obra de juventud y a Barbieri comenzar un trabajo parcial sobre el tema. Al aparecer la posibilidad de reponer Don Quijote en Sierra Morena en 1861, se les dio la ocasión a ambos para recuperar materiales que se habían quedado en el camino.

Barbieri colabora en la obra por indicación de Ventura de la Vega y lo hace gratis, tal como apunta el propio Barbieri, que cobra en especie “... acompañaba un obsequio que me hizo de un tintero, una pluma, cucbillo de cortar papel y sello, todo de madera tallada y construcción alemana, por haberle yo compuesto sin retribución alguna la música atrás indicada..." (Casares, 1994: 244) ${ }^{15}$. El Legado Barbieri nos aporta información de primera mano sobre el encargo quijotesco: “...El martes 23 de abril de 1861 la Real Academia Española celebró por primera vez el aniversario de la muerte de Cervantes con una función religiosa en la iglesia de las Trinitarias. En el mismo día el teatro del Príncipe hacía también una función conmemorativa en la cual se representaba la comedia D. Quijote de la Mancha que aunque rigurosamente no pertenece al género de la zarzuela, yo la incluyo en esta colección porque hice para ella la música del Ovillejo de Cervantes que canta Cardenio en el primer acto, un baile característico español para el segundo y una marcha y coro final para el acto tercero y cuya marcha heroica compuse sobre el motivo de las seguidillas manchegas..."(Casares, 1994: 281).

El hecho curioso es que la inclusión de la música de Barbieri en la obra de Ventura de la Vega no se hace con el texto propio sino con el original cervantino. Barbieri, al recuperar parte de lo que había compuesto tres años atrás, se limita a revisar la partitura con pequeños cambios que son visibles en el manuscrito original y que modifican solo parcialmente la idea primigenia. En la partitura original del Ovillejo no existe ni tan siquiera una referencia a la obra de Ventura de la Vega, usando únicamente la primera copla del Ovillejo original de Cervantes. La razón más plausible es que la composición se produjo de acuerdo con el libreto inconcluso de La Venta Encantada y no con Don Quijote en Sierra Morena. Este extremo se demuestra al analizar el manuscrito original de Barbieri, que presenta, con tipos de letras diferentes, la consignación de Música del maestro Barbieri, pero en ningún momento aparece referencia alguna a Ventura de la Vega, apareciendo escrito Letra de Cervantes. Sin duda es un hecho anecdótico, pero muy significativo a la hora de concluir que no existía una dirección ejecutiva clara en la representación de 1861 que unificase criterios.

\footnotetext{
13 Barbieri cita a Adolfo García en una extraña confusión entre nombre real y pseudónimo, pues se refiere a Gustavo Adolfo Bécquer, que firmó el libreto de La venta encantada en 1859 bajo el nombre de Gustavo García. La música de la zarzuela fue compuesta por el gaditano Antonio de Reparaz.

${ }^{14}$ Legado Barbieri, Ms. 14078, 317.

${ }^{15}$ Legado Barbieri, Ms. 14079.
} 


\section{CANTA CARDENIO, CANTA. UN OVILLEJO DE IDA Y VUELTA}

El Ovillejo de Barbieri aparece mediado el primer acto y se trata de una aria pequeña para tenor, pese a que la clave no aparece octavada, y cuerdas (violines, violas y violoncellos). Barbieri aprovechó el momento contemplativo de la escena subrayando enormemente la propia musicalidad de las palabras de Cardenio. El tipo de acompañamiento se presenta adecuado al carácter musical de la obra, introspectivo y nostálgico. Se presenta en un tempo Andante con un tipo de escritura de oficio donde el acompañamiento alla chitarra, se suele indicar con las señales de repetición en un claro ejercicio de oficio. Barbieri ubica su Ovillejo en la línea de las arias operísticas de la transición del Belcanto, deudor de dicha estética, pero claramente asentado en un sonido operístico, tal como afirmaba el propio compositor sobre su obra.

Barbieri elige el compás ternario de $6 / 8$ con una armadura de cuatro bemoles en tonalidad de fa menor. El caso de la elección de un tiempo ternario parece que abunda en un trabajo de número lento y contemplativo al estilo de las arias introductorias belcantistas. El uso de una tonalidad menor es evidentemente un recurso dramático esencial de acuerdo de las bases del drama italiano y la barroca teoría de los afectos que tanta influencia tiene en el despegue de la ópera romántica. El Ovillejo de Barbieri subraya por tanto una clara tendencia operística, no ya solo en los usos armónicos y texturas, sino también en el tipo de canto y de fraseo empleados.

La obra se desarrolla en 26 compases y, de acuerdo con el manuscrito original, en tres páginas apaisadas con tres sistemas cada una, excepto la última que tan solo presenta un sistema de tres compases con rúbrica final. No existen elementos que permitan pensar en una repetición del ovillejo con los textos de las otras coplas, si bien al ser un elemento inserto en una obra de teatro, quizá dicha repetición se daba por entendida. Lo cierto es que las versiones realizadas de la obra no contemplan esta posibilidad.

El trabajo armónico de Barbieri presenta un oficio importante, siendo un trabajo efectivo, aunque sin grandes novedades. Los violines asumen el trabajo de despliegue armónico mientras que los violonchelos simplemente presentan el bajo con un acorde tendido por parte de las violas. El tipo de escritura es rápida y efectiva, sin apenas detalles y con señales frecuentes de repetición de la misma figuración. Es por ello que destaca el tipo de escritura vocal. De grafía redondeada, atenta a marcar cualquier detalle, cada frase posee acentos de expresión y ligaduras de canto que ratifican a Barbieri como un gran conocedor de la voz humana, de sus inflexiones y de su fonética. Se observan algunos pentimenti a lápiz que marcarían algunas diferencias estructuras entre las frases, pero que finalmente quedan unificadas en la redacción final.

Formalmente la propia estructura del Ovillejo tiende a marcar una forma bipartita correspondiente con la copla y la redondilla. En el caso de Barbieri, existe una definición bipartita algo suavizada por el acompañamiento, pero no así por el aspecto de intencionalidad expresiva. El primer octosílabo con su pie quebrado presenta una línea descendente desde la dominante (Do) que termina con la tónica en tres compases. Idéntico proceso observamos en el segundo octosílabo. El tercero marca una diferencia que se corresponde con breve paseo modulante hacia la dominante Do que enlaza con la segunda sección correspondiente con la redondilla que presenta una frase con dos secciones que a 


\section{MARÍA ÁNGELES FERRER ForÉS Y JUAN FranCisCo DE DiOS HERNÁNDEZ}

su vez marcan el punto climático de la obra. La primera sección de la redondilla presenta como elemento definitorio el discurso de negra (acento) - corchea como estructura ternaria, que contrasta en la segunda parte con una línea más ondulante que nos devuelve a la tónica. La segunda sección (anacrusa del compás15), con las palabras: "Pues me matan..." presentan un descenso que tiene su parangón en el bajo armónico, más cromático, que se repite a la tercera para terminar en un lento ritardando.

\section{CONCLUSIONES}

Ventura de la Vega y su revisión romántica de las aventuras de Don Quijote en Sierra Morena aparece como telón de fondo en la creación de dos obras vocales basadas en textos semejantes, pero cuya validez define dos líneas paralelas que definen el nacionalismo. Comparten elementos comunes obvios, pero igualmente planteamientos que unifican una fuente de inspiración común y ambivalente por tanto. Los ovillejos de Juan Pedro Esnaola y Francisco Asenjo Barbieri desarrollan conceptos vocales semejantes en la transmisión de sentimientos fruto de tonalidades menores ${ }^{16}$ y el uso de tiempos ternarios. La resolución armónica no asume grandes riesgos, ya que se trata de obras compuestas y adecuadas a unos entornos estéticos muy definidos: el salón romántico en Esnaola; y la música escénica en un acto de homenaje público en Barbieri. Dos entornos por tanto en los que la audacia y experimentación formal o lingüística, difícilmente podrían justificarse.

Ambas se sitúan en una vía de definición nacionalista. En el caso de Esnaola con los condicionantes de una inevitable justificación cultural dentro de un entorno de definición cultural propia. Sus características son comunes en toda América y definen un nacionalista romántico no indigenista o folclorista que se suele obviar, pero que es importante delimitar. Por su parte, Barbieri mantiene su lucha por definir el canto escénico en lengua castellana como garante de la música nacional frente a la imitación de las formas sinfónicas germánicas o al uso de otras lenguas en la escena teatral.

En medio de todos ellos, Cervantes y su ovillejo musical que se convierte en un referente cultural que reúne e inspira cualquier definición nacional dentro del mundo hispanoparlante, situándose por encima de divisiones y constituyéndose como una fuente inagotable de identidades.

\footnotetext{
${ }^{16}$ Cabría incluir también en esta aseveración la Canción de Dorotea, obra compuesta por Ernesto Halffter para la película de Luis Gil Don Quijote de la Mancha, en la que Dorotea a voz sola canta los versos que le corresponden a Cardenio en el original. La obra fue compuesta por Halffter en 1940 y la revisó con inclusión del piano en 1947. No obstante la obra comparte con las románticas aquí analizadas su tonalidad menor (re menor) y el uso del tiempo ternario. Curiosamente Halffter tampoco usa las tres estrofas sino dos, en su caso la $1^{\mathrm{a}}$ y la $3^{\mathrm{a}}$ desde el original cervantino.
} 


\section{CANTA CARDENIO, CANTA. UN OVILLEJO DE IDA Y VUELTA}

\section{BIBLIOGRAFÍA}

Alatorre, A. (1990). "Perduración del "ovillejo cervantino"”. NFRH, XXXVIII, n², pp. 643-674. México: Centro de Estudio Lingüísticos y Literarios.

Barbieri, F. A. (1892). Discursos leídos ante la Real Academia en la recepción pública del Excmo. Sr. Don Francisco Asenjo Barbieri el día 13 de marzo de 1892. Madrid: Real Academia de Bellas Artes de San Fernando.

Benedetto, R. di (1987). Historia de la Música, El siglo XIX, 1ª parte, vol. 8. Madrid: Turner.

Casares, E. (1994). Francisco Asenjo Barbieri, 2. Escritos. Madrid: ICCMU.

Esnaola, J. P. (2009). Cuaderno de Música (1844) [estudio preliminar de Bernardo Illari]. La Plata: Instituto Cultural de la provincia de Buenos Aires.

Illari, B. (2010). "Esnaola contra Rosas". Revista Argentina de Musicologia, 11, pp. 33-73.

Lolo, B. (2008). "Don Quijote de la Mancha de Francisco Asenjo Barbieri y Ventura de la Vega en las conmemoraciones de la Real Academia de la Lengua de 1861". Tus obras los rincones de la tierra descubren, VI CIND AC, 1, pp. 391-404.

Rodríguez Sánchez de León, M. J. (2006). "Don Quijote de la Mancha en Sierra Morena, Tributo de Ventura de la Vega a Cervantes, Lope y Calderón”. Cuadernos de Ilustración y Romanticismo, 14, pp. 209-220.

Fecha de recepción: 10/12/2016

Fecha de aceptación: 18/02/2016 